\title{
Effective cross sections for stray light calculations in laser interferometry: application to LISA science interferometer
}

\author{
Marco Nardello, Michel Lintz
}

Marco Nardello, Michel Lintz, "Effective cross sections for stray light calculations in laser interferometry: application to LISA science interferometer," Proc. SPIE 11852, International Conference on Space Optics — ICSO 2020, 118523Q (11 June 2021); doi: 10.1117/12.2599618

SPIE Event: International Conference on Space Optics - ICSO 2021, 2021, Online Only 


\section{International Conference on Space Optics-ICSO 2020}

Virtual Conference

30 March-2 April 2021

Edited by Bruno Cugny, Zoran Sodnik, and Nikos Karafolas
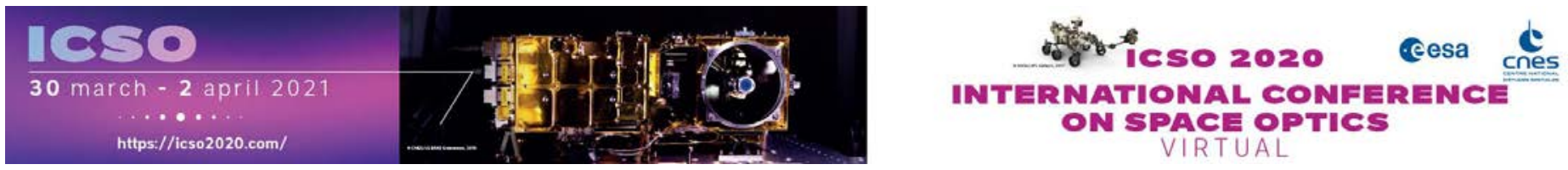

Effective cross sections for stray light calculations in laser
interferometry: application to LISA science interferometer

\section{Cesa issopocestings denes}




\title{
Effective cross sections for stray light calculations in laser interferometry. Application to the LISA science interferometer
}

\author{
Marco Nardello ${ }^{\mathrm{a}}$ and Michel Lintz ${ }^{\mathrm{a}}$ \\ aARTEMIS, OCA, Université Côte d'Azur and CNRS, 96 Boulevard de l'Observatoire, 06300 \\ Nice, France
}

\begin{abstract}
Interferometry is often used as a means for providing high sensitivity measurements. But interferometric measurements can also be easily affected by coherent stray light. In many fields, a correct estimation of the amount of stray light is necessary to assess the system performances and to guide the choice of the correct design. The LISA (Laser Interferometer Space Antenna) mission will consist of three spacecraft orbiting the Sun in a triangle constellation with arms of $2.5 \mathrm{Mkm}$ to detect gravitational waves. Detection is carried on thanks to two laser beams emitted by each spacecraft towards the two remote ones. A tiny fraction of the emitted beams is collected by the telescopes in the distant spacecrafts and interfered with the local laser beam, giving six heterodyne phase readouts which carry the imprint of the gravitational wave. Given the goal of reaching a noise floor of a few picometers on the distance measurement, this configuration will be very sensitive to stray light noise and for this reason stray light estimates are an important part of the design process. For a complex optical system stray light simulations can be made with specific optical analysis software (FRED, Zemax,...) which require expensive licences and computational time. To have a quick estimate one can try some manual calculation, but it is not always easy to find which scattering or reflection directions will lead to stray light able to couple into the interferometric measurement, not only with good superposition, but also with matching wavefront. We'll use the divergence of the laser beam to estimate the angular aperture under which a scattering element in the optical system can direct stray light into a detector, and show how these simple considerations can lead to stray light estimates which are in good agreement with the most advanced software simulations. These considerations are applicable to various optical systems.
\end{abstract}

Keywords: Stray light, interferometry, LISA, gravitational waves, scattering

\section{INTRODUCTION}

Interferometry is a powerful tool, which transforms very small optical path differences in measurable intensity variations. Today, thanks to the availability of monochromatic light sources (lasers) and the use of optical fibers and efficient photodetectors, interferometric measurements can be used in a variety of fields, such as distance, displacement, and vibration metrology; optical systems testing; surface topography; measurements of various parameters such as temperature, pressure, electrical and magnetic fields; spectroscopy. Among the recent applications are high-speed all-optical logic and the detection of gravitational waves 1.

The sensitivity of this technique makes it vulnerable to the presence of unwanted light in the system, in fact, the presence of $1 \%$ coherent stray light power can affect the measured intensity by up to $\pm 20 \%$, depending on the stray light optical phase. Stray light is due to scattering from roughness or contamination in the surfaces of the optical elements, or to unwanted reflections (ghosts) or diffraction. Since these are physical phenomena that cannot be avoided, it is important to anticipate the amount of stray light during the design phase, and act accordingly with appropriate technique to reduce it if necessary. Typical actions taken to reduce stray light are: modifying the optical design, moving surfaces away from stray light path or to make them less visible to the detector; insertion of baffles, to block stray light dangerous paths; painting or coating surfaces to reduce

Send correspondence to Marco Nardello

E-mail: marco.nardello@oca.eu, Telephone: +33 492003106 
reflectivity; cleaning surfaces to reduce contamination. None of these actions can eliminate stray light at $100 \%$, so the choice of the best course of action must be evaluated carefully 2 .

In the case of applications where high measurement precision is required, stray light control is a significant problem, as can be seen in publications from different fields, ranging from interferometric measurements for plasma control in fusion reactors 3 , to beam profile measurements in synchrothron facilities 4 , and interferometer performances in high precision formation flight 5,6. Gravitational waves detection, with the LIGO and Virgo interferometers, has opened a new page for observational astronomy, allowing the observation of binaries of neutron stars and black holes, events such as supernovae, and processes including those of the early universe shortly after the Big Bang. These instruments are constituted by Michelson interferometers with arms that are many kilometers long (effective optical path length in Virgo is $150 \mathrm{~km}$ ) and must be able to detect fluctuations of space in the order of the femtometer. These constraints make stray light a carefully considered issue 7.

LISA (Laser Interferometer Space Antenna) will take the challenge of gravitational wave detection to a new level. Scheduled for launch in 2034, it will bring to space three spacecraft, which will follow the Earth in its orbit around the Sun in a triangular formation with sides of 2.5 million kilometers. Lasers will be directed from one spacecraft to the others to obtain heterodyne interferometric measurements and monitor the passage of gravitational waves. The dimensions of the arms will allow to study gravitational waves at lower frequencies (from $0.1 \mathrm{mHz}$ to $1 \mathrm{~Hz}$ ) than those explored by LIGO and Virgo and thus obtaining information on new gravitational wave sources. Examples of LISA sources are supermassive black hole mergers, extreme mass ratio inspirals (i.e. a stellar object slowly spiralling towards a massive black hole), intermediate mass black hole binaries. Its ability to detect waves at low frequency will also allow for the monitoring of compact binaries in the Milky Way, weeks before the advent of their final merger, giving the opportunity for real-time observation of the electromagnetic or neutrino counterparts by providing the exact time and location of the event to the teams running the terrestrial LIGO and Virgo interferometers (Multi-messenger and multi-band gravitational wave astronomy). LISA will give also information on fundamental black hole physics, expansion of the universe and gravitational wave background 8 .

A comprehensive study of stray light is ongoing in the LISA community, to verify that the noise induced on the interferometers doesn't exceed the requirements 9. Many sources can bring potential problems in that respect, but one of the most critical is the telescope, since it will be used to collect approximately $700 \mathrm{pW}$ of the laser beam sent by the distant spacecraft, and simultaneously send the $1.5 \mathrm{~W}$ laser beam to the other spacecraft. In the baseline design of the LISA interferometry, the "Science Interferometer" implements the beating of the received beam with a pick-off of the emitted beam. But as the telescope backscatters a fraction of the emitted beam, the Science Interferometers can receive some of this backscattered light. The coupling of this stray, coherent light in the Science Interferometer can result in a shift of the effective optical phase of the reference beam, and so induce a systematic error in the interferometric phase read-out and a noise in the phase read-out, as soon as the structure that attaches the telescope to the interferometer undergoes fluctuations due to thermal expansion.

In this case, as in other instances previously cited, stray light will influence measurements if it is directed towards the detectors, but only if its overlap with the nominal beam is good enough, in position and direction, to affect interference. For complex optical system, understanding which is the correct cross section to be used in estimating stray light power is not a trivial task. For accurate results one can make use of specific software, like FRED, ASAP or Trace Pro, but it would be useful to be able to obtain fast estimates before committing to a more elaborate and expensive analysis. In this paper we present an approach based on physical and geometrical considerations that will serve to the purpose.

\section{SCATTERING FROM LISA TELESCOPE, ESTIMATION USING FRED SOFTWARE}

The LISA telescope is an afocal, off-axis design. It will be composed of 4 mirrors and will direct the gaussian beam entering through the small pupil of $2.24 \mathrm{~mm}$ towards the other spacecraft, after applying a magnification of 134. At the same time it will collect the flat top beam arriving from the other spacecraft with its acquisition field of $\pm 225 \mu \mathrm{rad} 10$. Fig. 1 shows the optical design. 


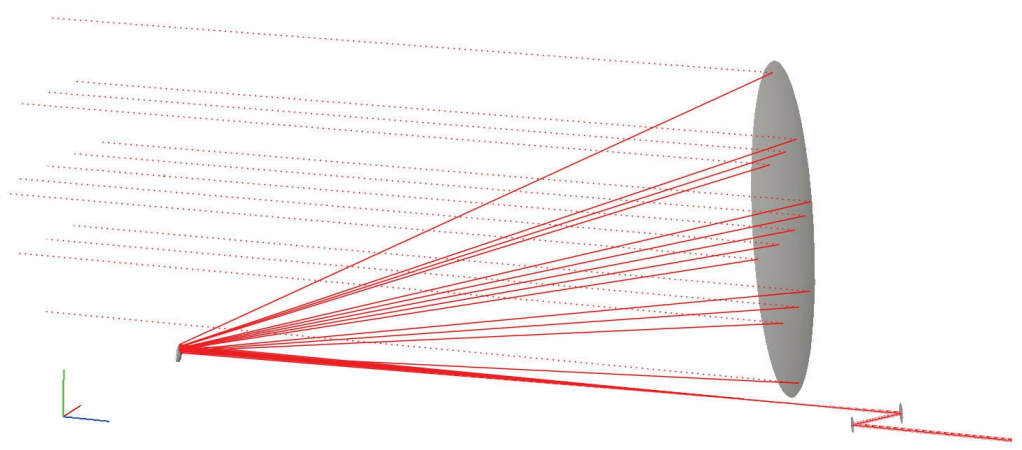

Figure 1. Telescope optical path: beam coming from space (left in the picture) arrives on the biggest ("primary") mirror and after 4 reflections is collimated onto the small pupil (right). Beam coming from the optical bench follows the inverse path and is directed to space.

The characteristics of the mirrors are listed in Table 1. Shown values are not final and have already suffered minor changes at the time of publication. This does not affect the conclusions of our work, which can be applied to different of optical systems

Table 1. Mirrors description.

\begin{tabular}{|l|l|l|}
\hline Mirror & Type & Clear aperture $(\mathrm{mm})$ \\
\hline M1 & Hyperbolic & 300 \\
\hline M2 & Even aspherical & 17.8 \\
\hline M3 & Elliptical & 20 \\
\hline M4 & Spherical & 16 \\
\hline
\end{tabular}

Backscattering is to be considered for the 4 optical elements. In the following, we will focus on scattering from roughness, showing which results software simulations give and how a simple, approximate but analytical, calculation allows us to arrive at compatible results.

The main parameter needed for simulation is roughness. Since these will be super-polished mirrors, for numerical application, we assume roughness of $2.5 \AA$ for $\mathrm{M} 1, \mathrm{M} 2$, M3 and $1 \AA$ for M4, since its spherical surface is more easily polished. FRED software uses the Harvey-Shack model to calculate BRDF (Bidirectional Reflectance Distribution Function), which is the differential ratio of scattered flux to the incident flux per differential projected solid angle:

$$
B R D F=\frac{d \Phi_{s} / \Omega_{s}}{d \Phi_{i} \cos \theta_{s}}
$$

To obtain BRDF values from roughness, Harvey-Shack models uses three parameters: $b_{0}, s, l$ which can be estimated based on the physical characteristics of the system or using previous experimental data 2. In the case of super-polished mirrors typical values are:

$$
\begin{gathered}
l=0.005 \\
s=-2.5 \\
b_{0}=0.004 s r^{-1}(\text { if roughness } 2.5 \AA) \\
b_{0}=0.026 s r^{-1}(\text { if roughness } 1 \AA)
\end{gathered}
$$

Once the parameters are chosen, the telescope optical setup can be uploaded in the analysis software, that will trace rays starting from the small pupil of the telescope through the system. When a ray will intercept one of 
the scattering surfaces, it will generate scattered rays in random directions, that can be collected by an analysis surface in correspondence of the entrance pupil. Only rays arriving on the pupil at the appropriate position and direction will be taken into account. Obtained results are shown in table 2 and are expressed as a ratio to the power entering the system.

Table 2. Telescope backscattered power ratios according to FRED simulations.

\begin{tabular}{|l|l|}
\hline Mirror & Backscattered power ratio \\
\hline M1 & $1.41 \cdot 10^{-16}$ \\
\hline M2 & $2.29 \cdot 10^{-13}$ \\
\hline M3 & $1.02 \cdot 10^{-12}$ \\
\hline M4 & $1.46 \cdot 10^{-13}$ \\
\hline
\end{tabular}

\section{APPROXIMATE, ANALYTICAL ESTIMATION}

To estimate back-scattered power without running numerical software simulations, one can use the formulas derived from Harvey-Shack theory and apply to them some physical and geometrical considerations. The two aspects that need to be solved are the calculation of the BRDF in the direction of interest, and the estimation of the effective solid angle under which the scattered rays overlap with the nominal beam. The second part is the one that appears not trivial.

\subsection{BRDF calculation}

BRDF is a function of the incidence angle $\theta_{i}$ and the scattering angle $\theta_{s}$. It can be calculated from the parameters shown in eq. 2 thanks to the formula:

$$
B R D F=b_{0}\left[1+\left(\frac{\left|\sin \theta_{s}-\sin \theta_{i}\right|}{l}\right)^{2}\right]^{\frac{s}{2}}
$$

Since we are interested in light that is backscattered towards the exit pupil, and with small angles compared to the direction of the original beam, the backscattered rays will have a $\theta_{s}=-\theta_{i}$. We can then write:

$$
B R D F=b_{0}\left[1+\left(\frac{\left|-2 \sin \theta_{i}\right|}{l}\right)^{2}\right]^{\frac{s}{2}}
$$

For this approximated calculation we consider all the power as concentrated on the ray propagating from the center of the exit pupil, so that we can deal with only one position and angle for each mirror instead of the actual ranges.

To calculate $\sin \theta_{i}$ for a chosen ray, having at our disposal positions and orientations of each mirror, we can use the ray transfer matrices and obtain ray position and direction at each interface: For reflection from a curved mirror:

$$
\left(\begin{array}{l}
y_{1} \\
\theta_{1}
\end{array}\right)=\left(\begin{array}{cc}
1 & 0 \\
-\frac{2}{R \cos \theta_{M}} & 1
\end{array}\right)\left(\begin{array}{l}
y_{2} \\
\theta_{2}
\end{array}\right)
$$

where $y_{1}$ and $\theta_{1}$ are ray position and angle before reflection, $R$ and $\theta_{M}$ are radius and inclination of mirror axes, and $y_{2}$ and $\theta_{2}$ are ray position and angle after reflection.

Results will be correct only in paraxial approximation. When this approximation is not valid, numerical simulation are needed to have accurate results. The values we found for incidence angles on each mirror are:

$$
\begin{aligned}
& \theta_{i(M 1)}=7.9^{\circ} \\
& \theta_{i(M 2)}=7.7^{\circ} \\
& \theta_{i(M 3)}=9.3^{\circ} \\
& \theta_{i(M 4)}=9.5^{\circ}
\end{aligned}
$$


Putting 6 and 2 in 4 we obtain:

$$
\begin{aligned}
& B R D F(M 1)=1.37 \cdot 10^{-6} \\
& B R D F(M 2)=1.46 \cdot 10^{-6} \\
& B R D F(M 3)=9.26 \cdot 10^{-7} \\
& B R D F(M 4)=1.40 \cdot 10^{-7}
\end{aligned}
$$

\subsection{Solid angle calculation}

We have calculated in the previous section the BRDF for rays scattered in the direction of the nominal beam $\left(\theta_{i}\right.$ in figure 2). Those rays will overlap exactly with the nominal beam and will alter the interferometry measurements. The next step is to consider rays scattered at an angle $\theta_{s}=\theta_{i}+\delta \theta$. Those rays will arrive on the small pupil at a certain angle $\theta$ from the nominal direction, and this angle $\theta$ will have a certain dependence on $\delta \theta$ that varies for each optical element in the system. The task we set to accomplish is to find the range of $\delta \theta$ values, for each optical element in the system, that will result in angles $\theta$ close enough to the nominal direction to cause interference.

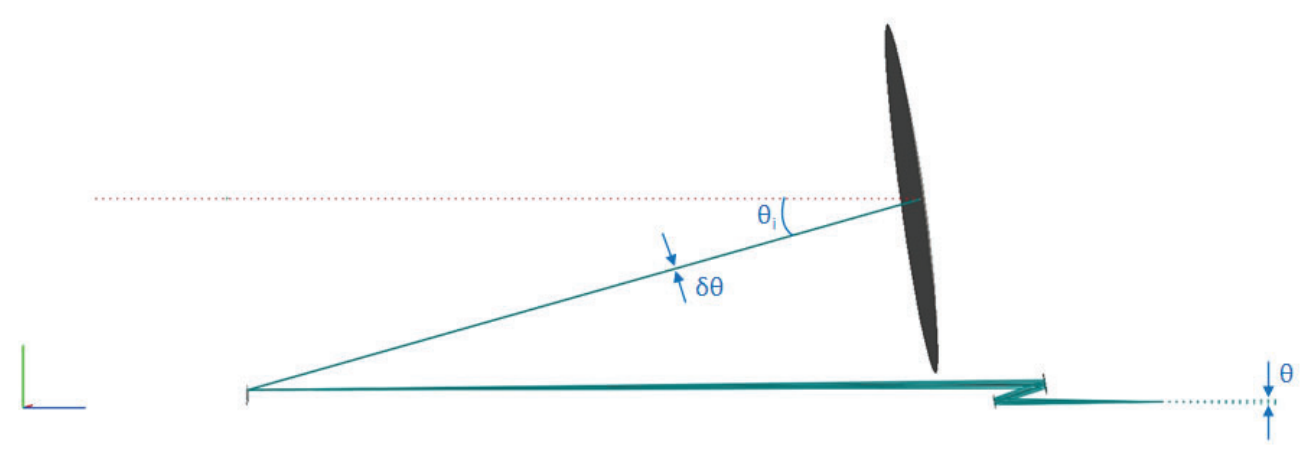

Figure 2. A nominal ray (dotted red) coming from the small pupil (right) is reflected by the 4 mirrors and exits the system to the left. Along its path it generates scattered rays (green) that are backscattered in a direction $\theta_{s}$ close to $\theta_{i}$ and that will return to the small pupil, entering it at an angle $\theta$ that will be related to the difference $\delta \theta$ between incidence and scattering angle.

This can be accomplished reasoning on geometrical considerations and physical behaviour of light. The procedure can be explained with the aid of the scheme of figure 3. Rays scattered (green) from optical element (1) are of interest if they overlap the nominal beam coming from the distant spacecraft and entering the local interferometer (orange). In the case of the LISA telescope, the nominal beam is a flat top beam. Since the optical elements are curved mirrors, they will focus the nominal beam towards (or away from) a focal point; because of diffraction, that point has a finite extension (A), due to the angular divergence given by the formula

$$
\sin \theta_{\text {Airy }}=1.22 \cdot \frac{\lambda}{D_{M}}
$$

where $D_{M}$ is the diameter of the focusing element (optical element (1) in the figure 3).

Since that divergence angle is the minimum angle necessary to resolve two different beams, scattered rays whose scattering direction differs from that of backscattering by an angle $\delta \theta \leq \theta_{\text {Airy }}$ cannot be resolved from the nominal beam and they will overlap and interfere. The argument is valid for every optical element in the system. 


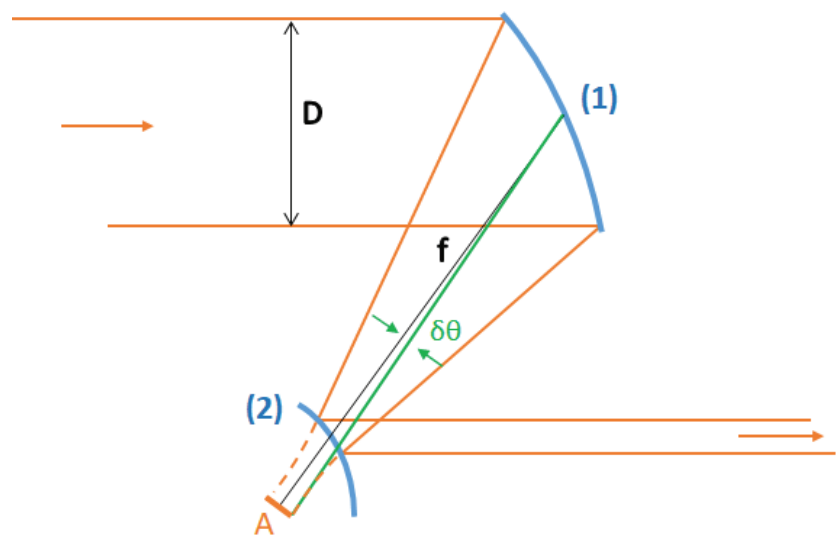

Figure 3. Schematics of concept for calculation of scattered rays angular acceptance.

The diameters used in 8 to calculate $\theta_{\text {Airy }}$ for each mirror are:

$$
\begin{gathered}
D_{(M 1)}=300 \mathrm{~mm} \\
D_{(M 2)}=8.2 \mathrm{~mm} \\
D_{(M 3)}=2 \mathrm{~mm} \\
D_{(M 4)}=2.24 \mathrm{~mm}
\end{gathered}
$$

From 8 we obtain:

$$
\begin{aligned}
\delta \theta_{(M 1)} & =4.33 \cdot 10^{-6} \mathrm{rad} \\
\delta \theta_{(M 2)} & =1.58 \cdot 10^{-4} \mathrm{rad} \\
\delta \theta_{(M 3)} & =6.49 \cdot 10^{-2} \mathrm{rad} \\
\delta \theta_{(M 4)} & =5.80 \cdot 10^{-2} \mathrm{rad}
\end{aligned}
$$

From that one can calculate the corresponding solid angles:

$$
\begin{aligned}
\Theta_{(M 1)} & =5.88 \cdot 10^{-11} s r \\
\Theta_{(M 2)} & =7.87 \cdot 10^{-8} s r \\
\Theta_{(M 3)} & =1.32 \cdot 10^{-6} s r \\
\Theta_{(M 4)} & =1.05 \cdot 10^{-6} s r
\end{aligned}
$$

Considering BRDF as a constant in these angular ranges, a simple multiplication between 7 and 11 gives the expected backscattering power (normalized to the incident power). We can compare the obtained numbers with the results given by the simulations, as shown in table 3 :

Results differ from simulated values by less than a factor 2; between the 4 mirrors, error is the greatest when the incidence angle varies the most along the mirror surface. In that case, in fact, the approximation we made of considering only the central ray becomes less optimal. The choice of the correct solid angle, which is the key part of this procedure, works correctly and results thus obtained can be used for cross-checking the output of numerical software simulations, or to obtain quick results in absence of dedicated software. In the example provided for the optical system (an afocal beam expander) the very large difference between the back-scattering from mirror M1 and from mirror M2 (more than three orders of magnitude) is correctly retrived by the approximate method, showing the effectiveness of the method.

\section{CONCLUSIONS}

In laser interferometry, coupled coherent stray light can limit the accuracy of the measurements. A simple, even if approximate, estimation of the amount of stray light coupled in the interferometers is a very useful tool for 
Table 3. Comparison between backscattering values obtained with analytical approach and values obtain with FRED simulations.

\begin{tabular}{|l|l|l|}
\hline Mirror & Simulation & Analytical calculation \\
\hline M1 & $1.41 \cdot 10^{-16}$ & $8.06 \cdot 10^{-17}$ \\
\hline M2 & $2.29 \cdot 10^{-13}$ & $1.15 \cdot 10^{-13}$ \\
\hline M3 & $1.02 \cdot 10^{-12}$ & $1.23 \cdot 10^{-12}$ \\
\hline M4 & $1.46 \cdot 10^{-13}$ & $1.48 \cdot 10^{-13}$ \\
\hline
\end{tabular}

stray light assessment. In this paper we show formulas and arguments that allow to understand, in terms of Airy patterns, how large is the fraction of scattered light that will be coupled into the interferometer. The method is general and applicable to many interferometric systems, its validity is shown here in the case of the stray light in the LISA mission, a critical aspect of the design of this interferometric gravitational wave observatory mission.

\section{ACKNOWLEDGMENTS}

The authors thank Jeffrey Livas for advice and for providing the telescope model.

\section{REFERENCES}

[1] Hariharan, P., [Basics of Interferometry], elsevier (2007 (second edition)).

[2] Fest, E. C., [stray Light Analysis and Control], Spie Press, Bellingham, Washington, USA (2013).

[3] Zou, Z. Y., Liu, H. Q., Ding, W. X., Chen, J., Brower, D. L., Lian, H., Wang, S. X., Li, W. M., Yao, Y., Zeng, L., and Jie, Y. X., "Effects of stray lights on faraday rotation measurement for polarimeter-interferometer system on east," Review of Scientific Instruments 89(1), 013510 (2018).

[4] Masaki, M. and Takano, S., "Two-dimensional visible synchrotron light interferometry for transverse beamprofile measurement at the SPring-8 storage ring," Journal of Synchrotron Radiation 10, 295-302 (Jul 2003).

[5] Noecker, C., Fleming, J., and Gorham, P., "Preliminary stray light analysis for the starlight mission," in [2001 IEEE Aerospace Conference Proceedings (Cat. No.01TH8542)], 3, 3/1445-3/1451 vol.3 (March 2001).

[6] Noecker, C., Wei, Z., and Decino, J., "Stray light estimates for the TPF formation flying interferometer," in [Optical Systems Degradation, Contamination, and Stray Light: Effects, Measurements, and Control], Fleming, J. C., Dittman, M. G., and Chen, P. T. C., eds., 5526, 249 - 256, International Society for Optics and Photonics, SPIE (2004).

[7] Bassan, M., [Advanced Interferometers and the Search for Gravitational Waves], Springer International Publishing (2014).

[8] Danzmann, K., "LISA mission L3 proposal." Submitted to ESA (2017).

[9] Sasso, C. P., Mana, G., and Mottini, S., "The LISA interferometer: impact of stray light on the phase of the heterodyne signal," Classical and Quantum Gravity 36, 075015 (mar 2019).

[10] Sankar, S. R. and Livas, J., "Optical alignment and wavefront error demonstration of a prototype LISA telescope," Classical and Quantum Gravity 37, 065005 (feb 2020). 